Polymer Journal, Vol. 38, No. 12, pp. 1245-1250 (2006)

(C)2006 The Society of Polymer Science, Japan

\title{
Preparation of Poly ( $n$-butyl acrylates) Encapsulated Carbon Black via Ultrasonic Irradiation Initiating Emulsion Polymerization
}

\author{
Qiuying LI, Guozhang WU, Xiliang ZHANG, and Chifei $\mathrm{WU}^{\dagger}$ \\ Polymer Alloy Lab., School of Material Science and Engineering, East China University \\ of Science \& Technology, 130 Meilong Road, Shanghai, P. R. China
}

(Received June 1, 2006; Accepted September 23, 2006; Published November 2, 2006)

\begin{abstract}
The encapsulation of poly ( $n$-butyl acrylates) (PBA) onto carbon black (CB) surface via ultrasonic irradiation initiating emulsion polymerization was investigated. The results showed that the route performed at ambient temperature can efficiently prepare PBA encapsulated $\mathrm{CB}$ as proved by the aggregation of PBA encapsulated $\mathrm{CB}$ in the precipitator of PBA. The surface properties of CB coated with PBA were analyzed by FT-IR and XPS. The content of PBA encapsulated on the CB surface reaches $11.87 \%$ determined by TGA. Compared with the conventional chemical modification of $\mathrm{CB}$, it was found that the particle size of $\mathrm{CB}$ obtained by the present approach can be controlled, and dynamic light scattering results quantified that $\mathrm{CB}$ particles with narrower size distribution and smaller size were obtained. The PBA encapsulated $\mathrm{CB}$ can be dispersed uniformly in the polymer matrix.
\end{abstract}

[doi:10.1295/polymj.PJ2006053]

KEY WORDS Carbon Black / Ultrasonic Irradiation / Emulsion Polymerization / Poly ( $n$-butyl acrylates) /

Carbon black (CB) is widely used in rubber, plastics, pains and inks that act as a reinforcing agent, black pigment, and electrically conductive filler. Although the primary particles of $\mathrm{CB}$ are known as nano-material, they are quite easy to fuse together to form aggregates and further to big agglomerates, which often result in the poorer mechanical properties of the filled polymer materials. So, the dispersion, compatibilization, and stabilization of CB in the polymer matrices are the key to develop the potential application of $\mathrm{CB}$ for functional composites. To solve these problems, many researchers have concentrated on the surface modification of CB. Approaches of the surface modification in the early include physical adsorption, heat treatment, oxidation, and plasma treatment. Grafting of polymers onto the surface of $\mathrm{CB}^{1-7}$ or encapsulating $\mathrm{CB}$ by heterophase polymerization, emulsion polymerization ${ }^{8,9}$ has been proved to be an effective method. However, all of these methods have somewhat disadvantages, respectively. For example, grafting of polymer onto $\mathrm{CB}$ surface required many complicate steps, involving the pretreatment of the $\mathrm{CB}$ surface to introduce functional groups. The rigorous pretreatment involved $\mathrm{HNO}_{3}$ oxidation is dangerous and unfriendly to environment. Recently, Wang ${ }^{10,11}$ reported a method of coacervation for encapsulating CB. Another significant advantageous of this route was the polymer encapsulated CB nanoparticles were free from aggregation except for without involving of complex polymerization. Although the method avoided the complicated polymerization, the process of ball milling to pulverize CB particles was involved and many organic solvents were added. So, it is of great interesting to develop a relatively simple polymerization technique for preparation of polymer encapsulated CB with controlled particles.

The application of ultrasonic irradiation in emulsion polymerization has been intensively investigated in recent years. ${ }^{12-17}$ Without involving any chemical initiators, the polymerization process can be performed at ambient temperature $\left(30-40{ }^{\circ} \mathrm{C}\right)$ due to the radicals formation arisen from the ultrasonic cavitation. Cavitation cause small voids in the liquid medium which periodically collapse leading to transient regions of high temperature of around $5000 \mathrm{~K}^{18}$ and pressure of about $500 \mathrm{~atm}$. Most of radicals are generated from the surfactant decomposition caused by the localized extreme conditions. ${ }^{16}$ The ultrasonic irradiation initiate some monomer such as methyl methacrylate, $n$-butyl acrylates and aniline in situ emulsion polymerization in the presence of nano materials included in multi-walled carbon nanotube, $\mathrm{SiO}_{2}, \mathrm{Al}_{2} \mathrm{O}_{3}$ and $\mathrm{TiO}_{2}$ have been studied in details by Wang and coworkers, ${ }^{19-22}$ in which nano materials were encapsulated by polymer and nano materials can be redispersed at the nano scale. So it is possible to use this ultrasonic irradiation initiating encapsulating emulsion polymerization to prepare polymer encapsulated $\mathrm{CB}$ and to our knowledge, there is no report on applying this technique to modify $\mathrm{CB}$.

${ }^{\dagger}$ To whom correspondence should be addressed (E-mail: wucf@ecust.edu.cn). 
The aim of the present research is to prepare polymer encapsulated CB by ultrasonic irradiation initiating emulsion polymerization. Scheme 1 shows the routine of this new study. By adopting the dispersion, crushing, activation and initiate effect of ultrasonic irradiation, the big agglomerates or aggregates of CB are partly broken down and vinyl monomer proceeds to polymerize, which finally formed a polymer coated CB with smaller particle size and uniform particle size distribution. The dispersibility of polymer encapsulated CB in polymer matrix is also presented.

\section{EXPERIMENTAL}

\section{Materials and Reagents}

CB used is Printex-XE2-B provided by Degussa Corp. The as-received CB was extracted by boiling toluene for $24 \mathrm{~h}$ in a Soxhlet extractor and dried in vacuum before use. $n$-Butyl acrylate (BA) was purified using $10 \% \mathrm{NaOH}$ and distilled water for three times in order to remove the inhibitor before being distilled under vacuum. The purified BA was stored in refrigerator at $5{ }^{\circ} \mathrm{C}$ for use. Sodium dodocane sulphonate (SDS) and cetyltrimethylammonium bromide (CTAB) were used as supplied. BR-106 is a kind of acrylic resin obtained from Mitubishi Rayon. Other reagents were used without any further purification.

\section{Preparation of PBA Encapsulated $C B$}

$1.0 \mathrm{~g} \mathrm{CB}, 4 \mathrm{~mL}$ BA and $96 \mathrm{~mL} 0.2 \%$ SDS aqueous solution was introduced firstly into the reaction vessel. Then the mixture in the reaction vessel was thoroughly deoxygenated by bubbling with nitrogen thermo stated at $40^{\circ} \mathrm{C}$. A JY92-IID (Shanghai xin zhi) sonifier with a titanium horn was used to deliver $22 \mathrm{kHz}$ ultrasonic wave to the reaction mixture. After $30 \mathrm{~min}$ of irradiation at $300 \mathrm{~W}$ power output under nitrogen, the samples were frosted in refrigerator for two days and then precipitated by methanol. Finally, the precipitated mixtures were filtered, washed, vacuum dried at $60^{\circ} \mathrm{C}$. Ultrasonic irradiation initiating emulsion polymerization in the absence of CB was also carried out. The experimental procedures were as the same as the above.

\section{Characterizations}

UV-vis absorption spectrophotometer (VARIAN Cary 500) was used to evaluate the dispersion of CB in surfactant aqueous solution by the determination of absorption at $267 \mathrm{~nm}$. Fourier transform infrared spectra (FT-IR) of the samples in $\mathrm{KBr}$ pellets or ATR were recorded on a Nicolet AVATAR 360 FT-IR spectrometer. X-Ray photoelectron spectroscopy (XPS) experiments were carried out on a RBD upgraded PHI-5000C ESCA system (Perkin Elmer) with
$\mathrm{Mg} \mathrm{K} \alpha$ radiation $(\mathrm{h} v=1253.6 \mathrm{eV}, 250 \mathrm{~W}, 14.0 \mathrm{kV})$. The base pressure of the analyzer chamber was about $5 \times 10^{-8} \mathrm{~Pa}$. The sample was directly pressed to a self-supported disk $(10 \times 10 \mathrm{~mm})$ and mounted on a sample holder then transferred into the analyzer chamber. Binding energies were calibrated by using the containment carbon $(\mathrm{C} 1 \mathrm{~s}=284.6 \mathrm{eV})$. Thermo gravimetric analysis (TGA) was used to measure the polymer content in the polymer encapsulated CB. It was analyzed under a heating rate of $10 \mathrm{~K} / \mathrm{min}$ from room temperature to $800^{\circ} \mathrm{C}$ and a dynamic nitrogen flow of $50 \mathrm{~cm}^{3} / \mathrm{min}$. The aggregate particle size of PBA encapsulated $\mathrm{CB}$ or $\mathrm{CB}$ was determined by dynamic light scattering (DLS). The measurements were carried out by NICOMPTM 380 ZLS attached with Zeta potential supplement. A He Ne argon ion laser was light source and the wavelength of the laser light was $639 \mathrm{~nm}$. The detection angle was $90^{\circ}$ and the temperature was set at $25^{\circ} \mathrm{C}$.

To investigate whether or not the PBA encapsulated on the surface of $\mathrm{CB}, 50 \mathrm{~mL}$ of ethanol added $0.005 \mathrm{~g}$ PBA encapsulated CB or CB was dispersed by ultrasonic cleaning wash for $10 \mathrm{~min}$. Then $2 \mathrm{~mL}$ dispersion diluted by $13 \mathrm{~mL}$ ethanol was centrifuged for $1 \mathrm{~min}$ and $2 \mathrm{~min}$ at $2000 \mathrm{rpm}$, respectively.

Dispersibility of PBA encapsulated CB in BR-106 polymer was also studied. $0.05 \mathrm{~g}$ PBA encapsulated $\mathrm{CB}$ or $\mathrm{CB}$ and $5 \mathrm{~g}$ BR-106 were introduced in $50 \mathrm{~mL}$ ethyl acetate. After dispersing by ultrasonic cleaning wash for $20 \mathrm{~min}$, a thin-film was prepared on glass substrates by casting methods. Then the optical microscope was used to observe the disperse state of PBA encapsulated CB or CB in BR-106 thin-film.

\section{RESULTS AND DISCUSSIONS}

\section{Determination of Surfactant for Polymerization}

Surfactant is a radical source necessary for the ultrasonic irradiation initiating emulsion polymerization. For systems dispersed the nanoparticls, surfactant bilayers called admicelles can be formed on the surface of the nanoparticles and is the main polymerization location when the concentration of the surfactant solution is below the critical micelle concentration $(\mathrm{cmc}){ }^{23,24}$ The monomer will diffuse into the admicelles and occurs to polymerize, which lead to the formation of polymer-coated nanoparticles. Therefore it is necessary to discuss the dispersion stability of $\mathrm{CB}$ in surfactant aqueous solution before ultrasonic irradiation initiating polymerization. Figure 1 showed the absorption dependence of time curves of $\mathrm{CB}$ in SDS and $C T A B$ aqueous solution in which the weight ratio of surfactant to $\mathrm{CB}$ is 0.4 . The higher the absorption is, the better the dispersion of CB is. It is seen that the absorption of CB in SDS is clearly higher than that 


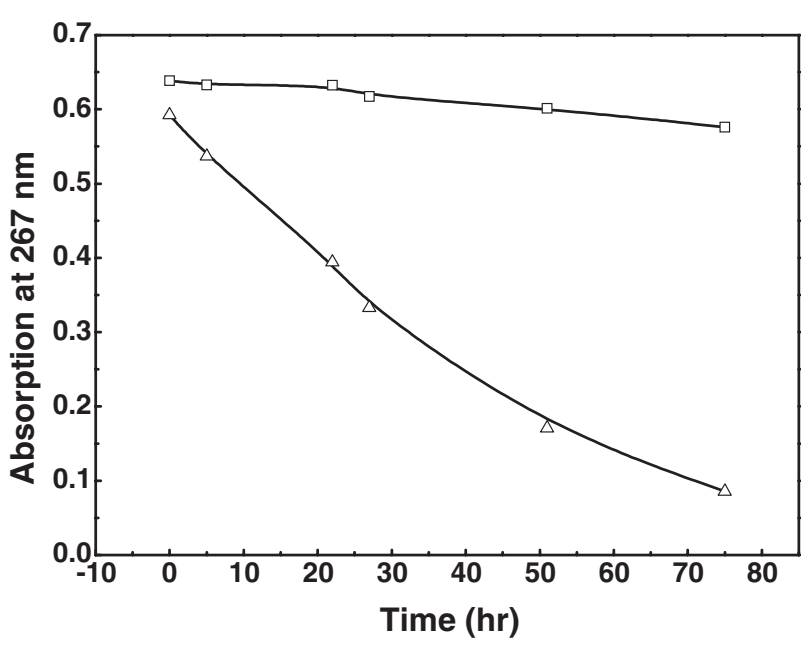

Figure 1. Absorption-time curves of CB in ( $\square$ ) SDS and $(\triangle)$ $\mathrm{CTAB}$ in $0.4 \%$ aqueous solution.

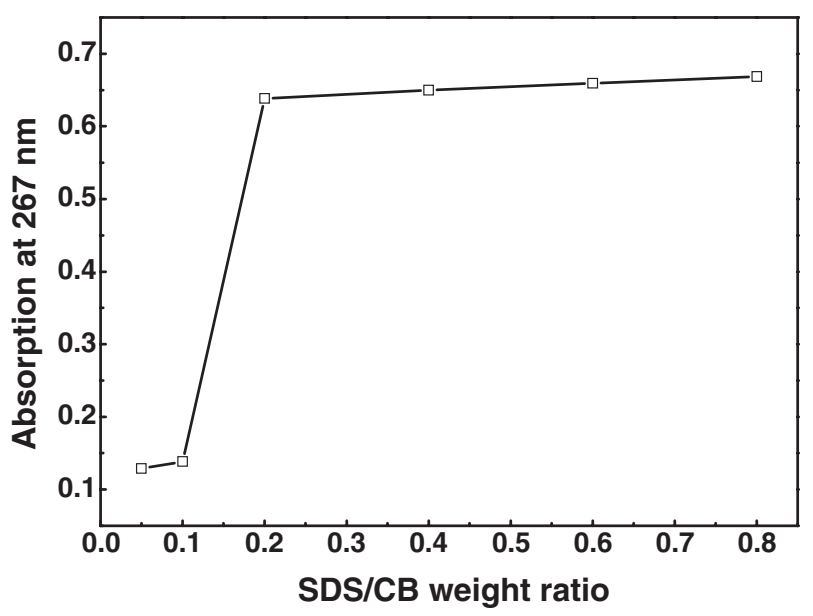

Figure 2. Effect of SDS/CB weight ratio on the dispersion of $\mathrm{CB}$.

of in CTAB and does not vary apparently with the time increasing, indicating that $\mathrm{CB}$ has better dispersion stability in SDS than in CTAB. The reason is that Zeta potential of the CB surface is positive and the $\mathrm{CB}$ surface is favorable to absorb anion surfactant SDS. Effect of SDS concentration on the dispersion of CB was further investigated, as shown in Figure 2. It can be seen that with the increasing of SDS concentration, the absorption at $267 \mathrm{~nm}$ increases. When SDS/ $\mathrm{CB}$ weight ratio reaches $0.2 / 1$, the absorption varies little, suggesting that the good dispersion is obtained. So in the following preparation of PBA encapsulated $\mathrm{CB}$ under ultrasonic irradiation, SDS surfactant in $0.2 \%$ of aqueous solution was chosen.

\section{Polymerization of PBA}

Although the ultrasonic irradiation initiating emulsion polymerization of $\mathrm{BA}$ has been reported detailed$1 y,{ }^{9}$ different ultrasonic horn and reaction conditions may result in different effects. As a systematic re-

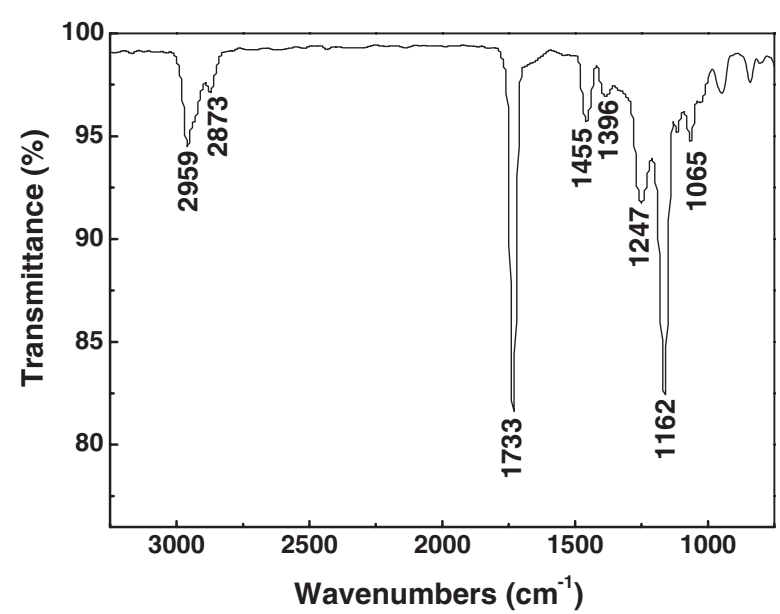

Figure 3. FT-IR spectra of PBA obtained from ultrasonic irradiation initiating emulsion polymerization of BA.

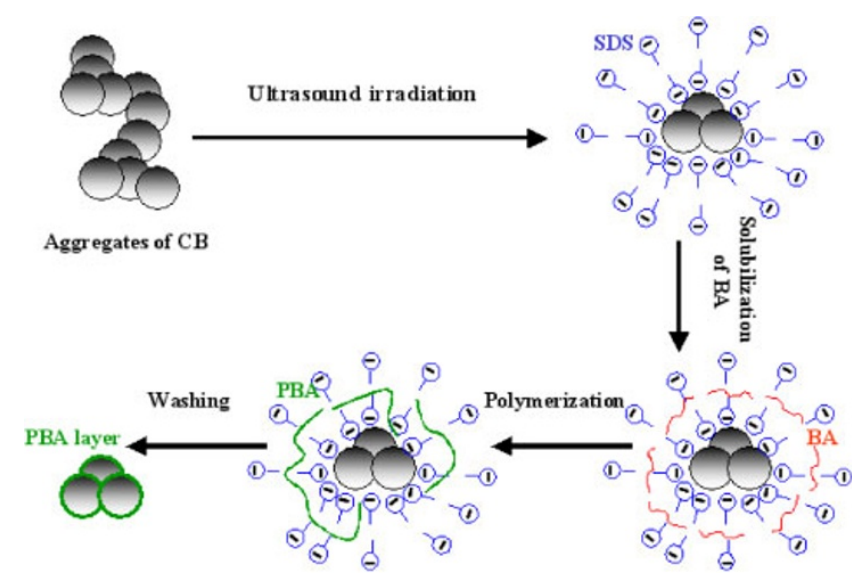

Scheme 1.

search, the ultrasonic irradiation initiating emulsion polymerization of BA in the absence of $\mathrm{CB}$ and chemical initiator requires to study. The FT-IR spectra of synthesized PBA shown in Figure 3 manifested that the characteristic peaks of the sample are consistent with that of standard PBA. The significant peaks at $1733 \mathrm{~cm}^{-1}$ corresponds to the $\mathrm{C}=\mathrm{O}$, and the peaks at $1000-1250 \mathrm{~cm}^{-1}$ suggest the presence of $\mathrm{C}-\mathrm{O}$ group. 2959 and $2873 \mathrm{~cm}^{-1}$ is assigned to the stretch vibration of $\mathrm{C}-\mathrm{H}$ bond. 1455 and $1396 \mathrm{~cm}^{-1}$ is attributed to the bend vibration of $-\mathrm{CH}_{2}$ or $-\mathrm{CH}_{3}$ group. These experiment results suggested that PBA could be synthesized by this method.

\section{Characterization of PBA Encapsulated CB}

As Scheme 1 shown, the preparation procedure of PBA encapsulated CB by ultrasonic irradiation initiating emulsion polymerization consists of four steps. Firstly the big agglomerates of $\mathrm{CB}$ were broken down into small aggregates and many new surface of $\mathrm{CB}$ particle were formed. Meanwhile, admicelle by the adsorption of the surfactant with different charge from 


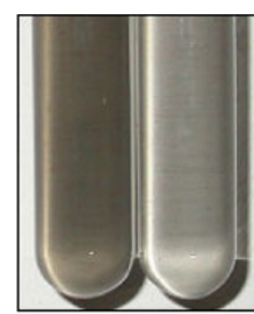

0 min.

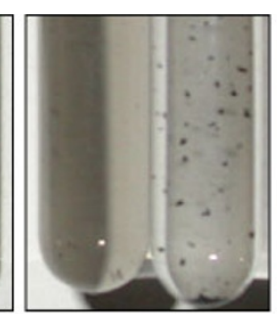

1 min.

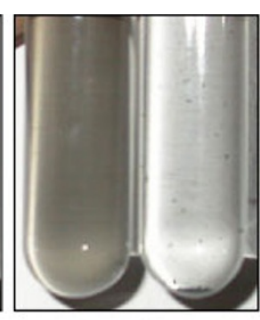

2 min.
Figure 4. Photographs of (left) CB and (right) PBA encapsulated $\mathrm{CB}$ suspension in ethanol at different centrifuged time.

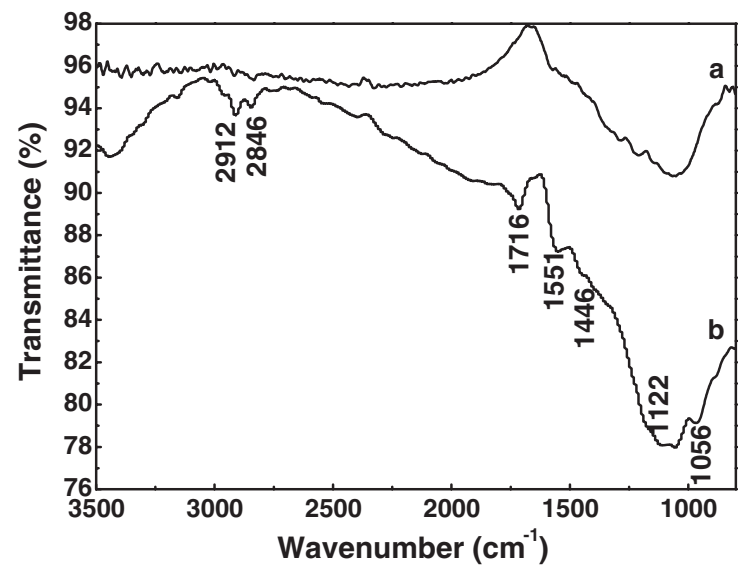

Figure 5. FT-IR spectra of (a) CB and (b) PBA encapsulated CB.

$\mathrm{CB}$ is formed onto the surface of $\mathrm{CB}$. In order to get as many as admicelle on the surface of $\mathrm{CB}$, surfactant concentration should be below the $\mathrm{cmc}$ and this also helped to prevent the competing of bulk polymerization. ${ }^{25}$ Secondly is the solubilization of BA into the admicelle. Then the polymerizations in the admicelle proceed. Because the surfactant concentration used here is below cmc the main polymerization location is in the admicelle adsorbed on the surface of CB. The last step is the washing to remove the surfactant using plenty of water. In order to check whether the CB has been coated by PBA or not we investigated the dispersion stability of the samples in ethanol. Because ethanol is the precipitator of PBA, if $\mathrm{CB}$ was encapsulated with PBA it will not disperse well and precipitate. As shown in Figure 4, PBA encapsulated $\mathrm{CB}$ begins to coagulate and precipitate to bottom with centrifugation time increasing, which verified that $\mathrm{CB}$ has been encapsulated by PBA.

Figure 5 showed FT-IR spectra of purified CB and PBA encapsulated CB prepared by ultrasonic irradiation initiating emulsion polymerization. It is obvious that there are no any absorption peaks in the spectra of $\mathrm{CB}$ because the $\mathrm{CB}$ used here has little functional groups. Furthermore, because of strong absorption of $\mathrm{CB}$, it is difficult to detect the few functional groups

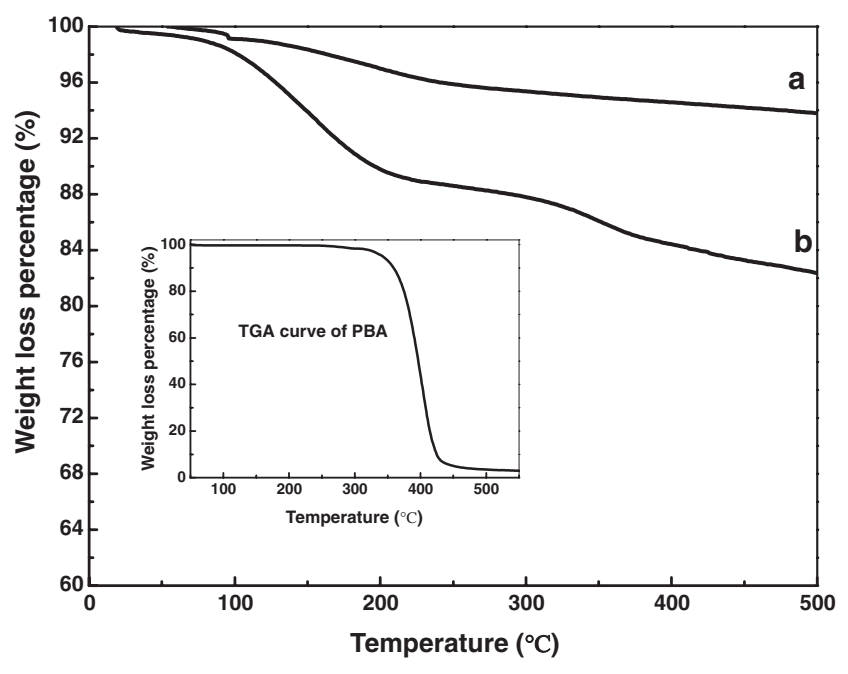

Figure 6. TGA curves of (a) $\mathrm{CB}$ and (b) PBA encapsulated CB.

onto the CB surface. For the FT-IR spectra of PBA encapsulated $\mathrm{CB}$, there appear new absorptions that are the characteristic peaks of PBA shown in Figure 3. The FT-IR difference between PBA and PBA encapsulated $\mathrm{CB}$ is the general shift of wavenumbers and the reason may arise from the interaction of $\mathrm{PBA}$ and $\mathrm{CB}$.

The exactly PBA content in PBA encapsulated CB can be calculated from TGA analysis, as shown in Figure 6. It appears two weight losses flat in the TGA curve for PBA encapsulated $\mathrm{CB}$ of which the higher weight loss flat from $250-500{ }^{\circ} \mathrm{C}$ is consistent with the decomposition of PBA (inserted in Figure 6) synthesized in the absence of $\mathrm{CB}$ and the corresponding content is $7.36 \%$. While the lower arises from on the one hand the oily matters contained in CB and the content is about $6.2 \%$ calculated from the TGA results of $\mathrm{CB}$. However, the weight loss lower than $250^{\circ} \mathrm{C}$ for PBA encapsulated $\mathrm{CB}$ is $10.71 \%$. So the rest $4.51 \%$ should be PBA that has different thermostability from PBA fabricated in the absence of CB.

XPS is another useful characterization method to identify the surface properties of $\mathrm{CB}$. The original $\mathrm{CB}$ and the PBA encapsulated $\mathrm{CB}$ were monitored by XPS. Figure 7 shows the XPS survey scan spectra of $\mathrm{CB}$ and PBA encapsulated CB. The figure shows the peaks corresponding to the $\mathrm{C}$ and $\mathrm{O}$ elements of which binding energies is 285 and 532, respectively. The high-resolution scan spectra of $\mathrm{C} 1 \mathrm{~s}$ and $\mathrm{O} 1 \mathrm{~s}$ region were shown in Figure 8 and the corresponding element atomic percent concentrations at the surface were listed in Table I. As can be seen from Table I, the increasing of $\mathrm{O} / \mathrm{C}$ atom ratio in the PBA encapsulated $\mathrm{CB}$ compared with $\mathrm{CB}$ further identifies the presence of PBA on the surface of CB. Although the changes in atomic concentration were in the direction expected for the encapsulation of the PBA, the ratio of 


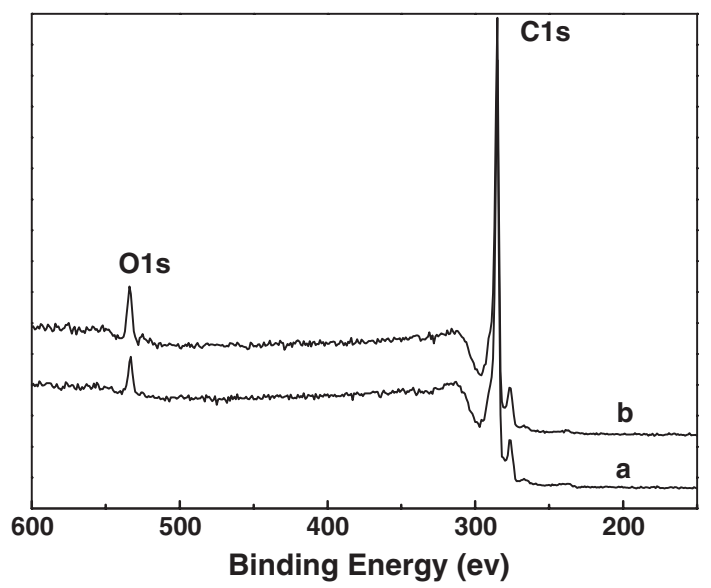

Table I. The carbon and oxygen content of $\mathrm{CB}$ and PBA encapsulated $\mathrm{CB}$

\begin{tabular}{ccc}
\hline & CB $(\%)$ & PBA encapsulated CB $(\%)$ \\
\hline $\mathrm{O}$ & 3.87 & 6.54 \\
$\mathrm{C}$ & 96.05 & 93.38 \\
\hline
\end{tabular}

Table II. Diameter of CB and PBA encapsulated CB particles

\begin{tabular}{ccc}
\hline & $\begin{array}{c}\text { Average particle } \\
\text { size }(\mathrm{nm})\end{array}$ & $\begin{array}{c}\text { Standard } \\
\text { deviation }(\%)\end{array}$ \\
\hline $\mathrm{CB}$ & 247.6 & 47.10 \\
PBA encapsulated CB & 169.0 & 43.60 \\
\hline
\end{tabular}

Figure 7. XPS survey scan spectrum of (a) CB and (b) PBA encapsulated CB.
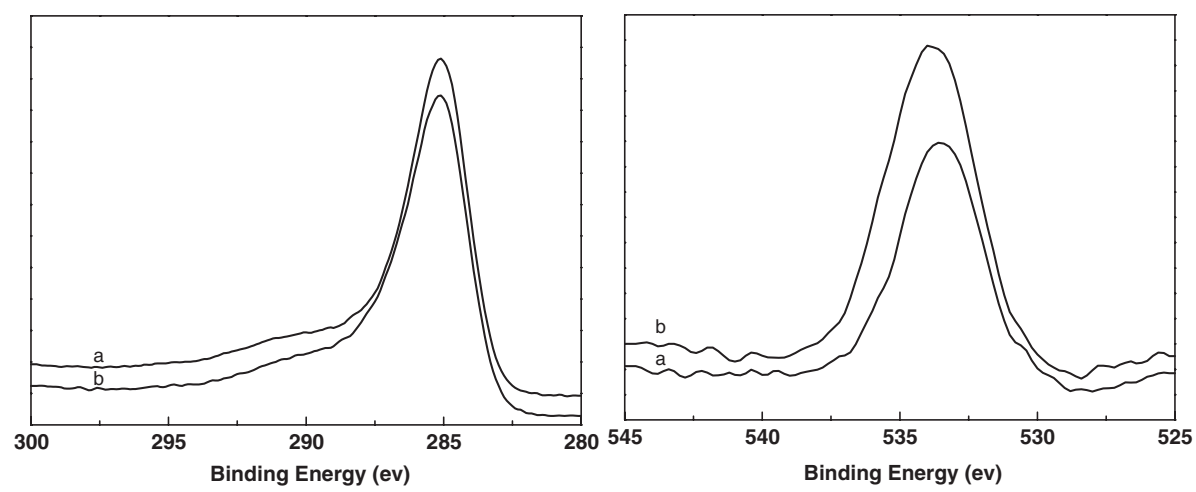

Figure 8. High-resolution (left) C1s and (right) O1s XPS spectra of (a) CB and (b) PBA encapsulated CB.

the oxygen and carbon determined from XPS is not 2:7, which should correspond to the PBA; instead it is 2:28.6. There is still much more carbon which does not originate from the PBA. This indicates that the surface coverage by the PBA is incomplete.

Ultrasonic irradiation can not only initiate polymerization to prepare polymer encapsulated $\mathrm{CB}$, but crush the agglomerates of $\mathrm{CB}$ and form small aggregates. This fact has been proved by DLS analysis result, as shown in Table II. DLS provides the information on the aggregate size of $\mathrm{CB}$. The $\mathrm{CB}$ particles have a diameter of $247.6 \mathrm{~nm}$ while the particle diameter of PBA encapsulated CB is $169.0 \mathrm{~nm}$ decreased $31.8 \%$ compared to $\mathrm{CB}$ particle size. The difference in the particle size between $\mathrm{CB}$ and PBA encapsulated CB is the outcome of the ultrasonic crushing effect produced from turbulent flow and shock waves during bubble collapse. ${ }^{26}$ The turbulent flow and intensive shock waves induce the high-speed interparticle collisions and the agglomerates and bigger aggregates are crushed into smaller aggregates. Noted that the standard deviation of PBA encapsulated CB is $43.60 \%$ also smaller than that of $\mathrm{CB}$. The lower the standard deviation is, the more uniform the particle size distri- bution is. It was concluded from the above results that $\mathrm{CB}$ with the smaller particle size and uniform particle size distribution could be obtained by the unusual sonochemical effect, which could not be achieved by the other conventional chemical encapsulated CB methods.

\section{Dispersibility of PBA Encapsulated CB in BR-106 Polymer}

The dispersibility of PBA encapsulated CB in BR106 polymer matrix was also examined. The thin-film prepared by PBA encapsulated CB/BR-106 and CB/ BR-106 was shown in the photograph inserted in Figure 9. Clearly, compared to CB/BR-106 thin-film, PBA encapsulated CB/BR-106 thin-film is much more flatness, suggesting that PBA encapsulated CB has better compatibility with BR-106 polymer. Optical microscope images (Figure 9b) show the particle size distribution of PBA encapsulated CB in BR-106 is more uniform and not congregate into agglomerates like CB in BR-106 (Figure 9a). This result evidenced the DLS results. The fact that the dispersion of PBA encapsulated CB in BR-106 has been improved may be attributed to ultrasonic irradiation make the $\mathrm{CB}$ 

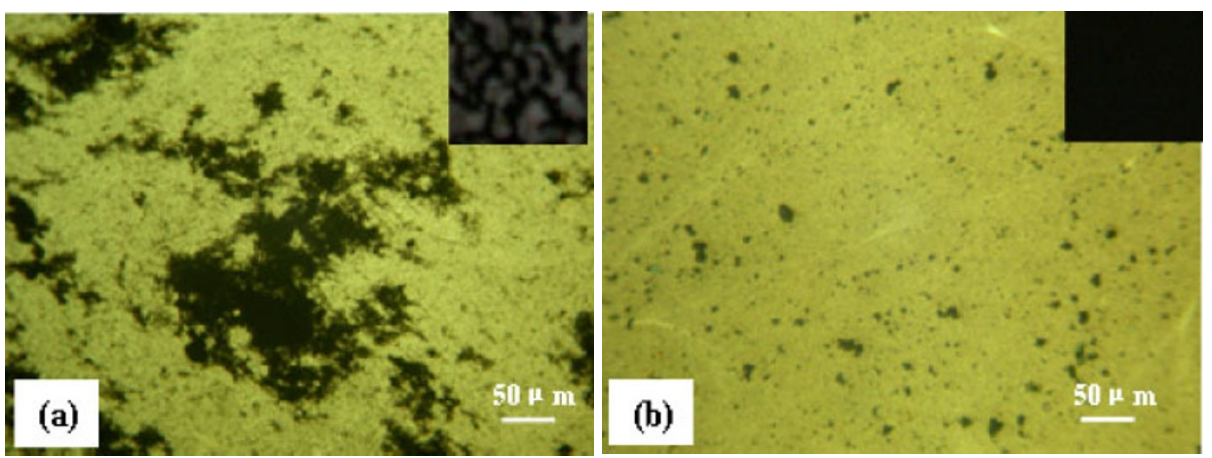

Figure 9. Optical microscope images of the thin-film of (a) CB/BR-106 and (b) PBA encapsulated CB/BR-106.

agglomerates crush into smaller aggregates and the PBA layer introduced on the surface of $\mathrm{CB}$ overcome the congregation of $\mathrm{CB}$ when filled in polymer matrix.

The purpose to encapsulate polymer to $\mathrm{CB}$ is to resolve the problems of dispersion, compatibilization and stabilization of CB in the polymer matrices, and further to endow with functionality of the corresponding composites. As the $\mathrm{CB}$ used in this present study is a kind of conductive $\mathrm{CB}$, the electric conductivity of the PBA encapsulated CB/BR-106 thin-film was studied. The primary results showed that the surface resistance of film filled with PBA encapsulated CB is a little higher than that of film filled with non-modified $\mathrm{CB}$ when the content is $20 \mathrm{wt} \%$. A large number of publications has shown that the conductivity of $\mathrm{CB} /$ polymer composite depends on many factors, such as $\mathrm{CB}$ content, the dispersing extent of the aggregate of $\mathrm{CB}$, the aggregate size and aggregate size distribution of $\mathrm{CB}$ etc. So, it is necessary to further discuss the application of PBA encapsulated CB in the conductive composites in the further work.

\section{CONCLUSTIONS}

The preparation of PBA encapsulated CB via ultrasonic irradiation initiating encapsulating emulsion polymerization provide an alternative route for the modification of CB. The preliminary results indicated that this route could generate efficient polymerization modifying in a rapid reaction at relatively low temperature. In addition, one of the most advantages of this method is to get uniform particle size distribution. These points made this preparation method for $\mathrm{CB}$ modification worth investigating further.

\section{REFERENCES}

1. N. Tsubokawa, Prog. Polym. Sci., 17, 471 (1992).

2. K. Fujiki, N. Tsubokawa, and Y. Sone, Polym. J., 22, 661 (1990).

3. N. Tsubokawa, K. Fujiki, and Y. Sone, Polym. J., 20, 213 (1988).
4. N. Tsubokawa, A. Yamada, and Y. Sone, Polym. J., 16, 333 (1984).

5. S. Hayashi and N. Tsubokawa, J. Macromol. Sci., Part A: Pure Appl. Chem., 35, 1781 (1998).

6. N. Tsubokawa, H. Ichioka, T. Satoh, S. Hayashi, and K. Fujiki, React. Funct. Polym., 37, 75 (1998).

7. K. Fujiki, M. Sakamoto, S. Yoshikawa, T. Satoh, and N. Tsubokawa, Compos. Interfaces, 6, 215 (1999).

8. F. Tiarks, K. Landfester, and M. Antonietti, Macromol. Chem. Phys., 202, 51 (2001).

9. N. Bechthold, F. Tiarks, M. Willert, K. Landfester, and M. Antonietti, Macromol. Symp., 151, 549 (2000).

10. H. Y. Li, H. Z. Chen, W. J. Xu, F. Yuan, J. R. Wang, and M. Wang, Colloids Surf., A, 254, 173 (2005).

11. H. Y. Li, H. Z. Chen, J. Z. Sun, J. Cao, Z. L. Yang, and M. Wang, Macromol. Rapid Commun., 24, 715 (2003).

12. S. Biggs and F. Grieser, Macromolecules, 28, 4877 (1995).

13. G. Cooper, F. Grieser, and S. Biggs, J. Colloid Interface Sci., 184, 52 (1996).

14. H. C. Chou and J. O. Stoffer, J. Appl. Polym. Sci., 72, 797 (1999).

15. Y. B. Liu, H. C. Chou, and J. O. Stoffer, J. Appl. Polym. Sci., 53, 247 (1994).

16. H. C. Chou and J. O. Stoffer, J. Appl. Polym. Sci., 72, 827 (1999).

17. Y. Q. Liao, Q. Wang, H. S. Xia, X. Xu, S. M. Baxter, R. V. Slone, S. G. Wu, G. Swift, and D. G. Westmoreland, J. Polym. Sci., Part A: Polym. Chem., 39, 3356 (2001).

18. K. S. Suslick, Science, 247, 1439 (1990).

19. H. S. Xia, Q. Wang, and G. H. Qiu, Chem. Mater., 15, 3879 (2003).

20. H. S. Xia, C. H. Zhang, and Q. Wang, J. Appl. Polym. Sci., 80, 1130 (2001).

21. H. S. Xia and Q. Wang, Chem. Mater., 14, 2158 (2002).

22. Q. Wang, H. S. Xia, and C. H. Zhang, J. Appl. Polym. Sci., 80, 1478 (2001).

23. J. Wu, J. H. Harwell, and E. A. O'Rear, Langmuir, 3, 531 (1987).

24. J. H. O'Haver, J. H. Harwell, and E. A. O'Rear, Langmuir, 10, 2588 (1994).

25. J. Wu, H. J. Harwell, and E. A. O'Rear, J. Phys. Chem., 91, 623 (1987).

26. B. Gompf, R. Gunther, G. Nick, R. Pecha, and R. Eisenmenger, Phys. Rev. Lett., 79, 1405 (1997). 